\title{
Entanglement, recoherence and information flow in an accelerated detector - quantum field system: Implications for black hole information issue
}

\author{
Shih-Yuin Lin* \\ Physics Division, National Center for Theoretical Sciences, P.O. Box 2-131, Hsinchu 30013, Taiwan \\ B. L. $\mathrm{Hu} u^{\dagger}$ \\ Joint Quantum Institute and Maryland Center for Fundamental Physics, \\ Department of Physics, University of Maryland, College Park, Maryland 20742-4111, USA and \\ Perimeter Institute for Theoretical Physics, 31 Caroline Street North, Waterloo, Ontario N2L 2 Y5 Canada
}

(Dated: April 26, 2008)

\begin{abstract}
We study an exactly solvable model where an uniformly accelerated detector is linearly coupled to a massless scalar field initially in the Minkowski vacuum. Using the exact correlation functions obtained in Refs. [1, 2] we show that as soon as the coupling is switched on one can see information flowing from the detector to the field and propagating with the radiation into null infinity. By expressing the reduced density matrix of the detector in terms of the two-point functions, we calculate the purity function in the detector and study the evolution of quantum entanglement between the detector and the field. Only in the ultraweak coupling regime could some degree of recoherence in the detector appear at late times, but never in full restoration. We explicitly show that under the most general conditions the detector never recovers its quantum coherence and the entanglement between the detector and the field remains large at late times. To the extent this model can be used as an analog to the system of a black hole interacting with a quantum field, our result seems to suggest in the prevalent non-Markovian regime, assuming unitarity for the combined system, that black hole information is not lost but transferred to the quantum field degrees of freedom. Our combined system will evolve into a highly entangled state between a remnant of large area (in Bekenstein's black hole atom analog) without any information of its initial state, and the quantum field, now imbued with complex information content not-so-easily retrievable by a local observer.
\end{abstract}

PACS numbers: 04.62.+v, 42.50.Lc 04.70.Dy

\section{INTRODUCTION}

Recent years saw increased interest and activities in understanding the nature and dynamics of entanglement in quantum systems. This is a distinctly quantum feature absent in classical systems, also the most essential factor in demonstrating the capability of any proposed quantum computer scheme. Many theoretical studies of quantum entanglement have been carried out for two level systems in atoms, quantum dots and superconductors with charges, spins and fluxes. Entanglement between qubits (quantum two-level systems) as mediated by a quantum fields is also of interest because of realistic experimental needs. This aspect has been considered by one of us and collaborators in popular models such as atoms in a cavity field (see [3, 4, 5] for two qubits in two separate fields and 6, 7, 8, 9, 10, 11] in one quantum field) and two Brownian oscillators in a finite temperature bath (see, e.g., 12, 13] and references therein). Associated with the dynamics of entanglement are the older issues of quantum decoherence and entropy / information evolution. Entanglement between two qubits is measured effectively by the concurrence functions [14], the entropy of formation, fidelity and purity (see, e.g., [15, 16]). Investigation of these issues are pursued most actively today in the area of quantum information.

Another area where questions of quantum entanglement, entropy and information are raised is in black hole physics and quantum gravity, where fundamental issues about the laws of physics and the structure of spacetime are of interest. Bekenstein's proposal of black hole entropy [17, 18] and Hawking's discovery [19, 20, 21] that black hole emits thermal radiance raise the question of how the end state of a black hole is like, and ushered in the debate whether there is net information lost in a black hole 22, 23, 24] (see, e.g., 25, 26] for an overview) and, by proxy, whether unitarity in the laws of physics is violated. There are many proposed explanations - remnants, naked singularity, baby universe

\footnotetext{
*Electronic address: sylin@phys.cts.nthu.edu.tw
}

†Electronic address: blhu@umd.edu 
formation or complete evaporation. (See e.g., [27, 28, 29, 30, 31, 32, 33].) We will focus on one explanation which bears on some of the issues in quantum coherence and information mentioned above, namely, that information in the black hole is not lost but transferred and dispersed into the quantum field through Hawking radiation. Many authors have proposed or hinted on this view, approached from different angles, some more explicitly than others, ranging from quantum gravity, string theory, nonequilibrium statistical mechanics and quantum information. Out of these we shall focus on the themes of two papers [34, 35] (which are shared in varying degrees by other authors, see papers cited above). For some recent discussions with newer perspectives, see e.g., [36, 37] from string theory and nonlocality viewpoints, 38] from loop quantum gravity and 39, 40] for a quantum information approach to this issue.

In Ref. 34] Anglin et al. studied a simple model of a harmonic oscillator coupled to a quantum field and analyzed the decoherence and recoherence of the detector in the ultraweak coupling approximation. They claimed that, as soon as the coupling is switched on, the oscillator loses the quantum coherence on a very short decoherence time corresponding to the cut-off time scale. But all the quantum coherence will recover in the end, after a much longer relaxation time. The initial information in the oscillator eventually flow into the field side, though appearing in a highly nontrivial form. They suggest that black hole information could behave in a similar way. In Ref. [35] one of us described a scenario where information in the black hole is transferred to the quantum field, and proposed to use the correlation functions of an interacting field as registers of information and the dynamics of correlations as a measure of information flow. According to the viewpoint put forth in [35], the appearance of information loss is primarily owing to the fact that actual physical measurements are limited in accuracy, i.e., one can only access the lowest order correlation functions, beginning with the mean field and the two-point functions. It also highlights the huge capacity of a quantum field in storing and dispensing information.

In our present work we adopt some modern methods of quantum information to address some aspects of black hole information issues. Looking at a particle detector in uniform acceleration interacting with a quantum field, we study the quantum entanglement, quantum recoherence and information flow in this simple system with the aim of providing an illustrative example to check on the ideas put forth in [35]. Of the main points made there, we can confirm that information does flow from the detector to the field and gets propagated to null infinity with the radiation. Further, from the behavior of two-point functions obtained in [1, 2], we do not see any qualitative change between the $a=0$ static case and the $a \neq 0$ uniformly accelerated cases. Insofar as the information behavior is concerned the uniformly accelerated cases are closer in nature to the inertial case than those cases with non-uniform acceleration [41, 42]. We also analyze the cases similar to those considered in [34] but with a much wider parameter range. We clarify that, in the ultraweak coupling limit, some level of quantum coherence is recovered at late times, but full recoherence is impossible as long as the coupling is on; Beyond the ultraweak coupling limit, late-time recoherence never occurs. In this study we show that with new tools adopted from quantum information, even simple models like this can provide valuable insight into deeper issues in statistical mechanical properties of detector (atom)- field interactions and by suitable extension, black hole spacetime physics.

This paper is organized as follows: In Sec. II we introduce a system with the uniformly accelerated detector (atom) coupled with the field. Using the exact form of the two-point functions we demonstrate that already at this stage one can see information flowing from the detector to the field and propagates with the radiation into null infinity. In Sec IIA we introduce a quantity called purity and show that it is useful for describing the entanglement and the recoherence dynamics of the system. To obtain the purity function we calculate the reduced density matrix of the detector in Sec. IIB. Then in Sec.III we derive analytic expressions for the purity in the detector and deduce the entanglement between the detector and the field, first from the detector's view (time) and then from the field's view (time). This behavior is illustrated with graphs plotted in various parameter ranges. In Sec. IV we make some technical remarks and then give a discussion of the central issues raised here. As a complementary description, in Appendix A, we give the reduced density matrix of the field for the same initial state used in Sec. II. For a detector initially in the ground state the purity of the detector and the purity of the field have simple enough expressions that we can record them in Appendix B. Finally in Appendix C we provide a comparison of our results to the claims of [34] on the recoherence issue.

\section{INFORMATION FLOW BETWEEN THE DETECTOR AND THE QUANTUM FIELD}

Consider a harmonic oscillator with bare mass $m_{0}$, bare natural frequency $\Omega_{0}$ and internal degree of freedom $Q$ (such as the Unruh-DeWitt(UD) detector [43, 44, 45] in the language of quantum field theory in curved spacetime) interacting with a massless quantum scalar field $\Phi$ in four-dimensional Minkowski space with coupling constant $\lambda_{0}$. The action of the combined particle detector - quantum field system is given by [1]

$$
S=\int d \tau \frac{m_{0}}{2}\left[\left(\partial_{\tau} Q\right)^{2}-\Omega_{0}^{2} Q^{2}\right]-\int d^{4} x \frac{1}{2} \partial_{\mu} \Phi \partial^{\mu} \Phi
$$




$$
+\lambda_{0} \int d \tau \int d^{4} x Q(\tau) \Phi(x) \delta^{4}\left(x^{\mu}-z^{\mu}(\tau)\right) .
$$

We will consider the cases when it is uniformly accelerated along the trajectory $z^{\mu}(\tau)=\left(a^{-1} \sinh a \tau, a^{-1} \cosh a \tau, 0,0\right)$ with proper acceleration $a$. For the cases of detectors at rest $(a=0)$, we have learnt in [1, 2] that the two-point functions of our UD detector theory in (3+1)D with finite $a$ have no singular behavior as $a \rightarrow 0$. Hence all our results below, which are expressed in terms of these two-point functions, apply equally well to the case of detectors at rest.

We study the case when the initial state of the combined system is a direct product of a quantum state $|q\rangle$ for the detector $Q$ and the Minkowski vacuum $\left|0_{M}\right\rangle$ for the field $\Phi$,

$$
\left|\psi\left(\tau_{0}\right)\right\rangle=|q\rangle \otimes\left|0_{M}\right\rangle \text {. }
$$

Oftentimes one hears the comment that since the detector is a harmonic oscillator and the field can be decomposed as a collection of harmonic oscillators, the combined system is just a system of $(\infty+1)$ oscillators. Indeed one can diagonalize the UD detector-field system into a system of $(\infty+1)$ free harmonic oscillators [34]. But we want to add the warning that the choice of initial conditions for the detector $Q$ (such as the ground state) and for the field $\Phi$ (such as the Minkowski vacuum) separately renders the set-up of this system (physical variables and initial states) physically different from that of the system of $(\infty+1)$ free harmonic oscillators with choices of the initial states deemed natural for it.

Since the combined system is linear, the operators evolve in the Heisenberg picture as linear transformations. For example, the operator of $Q$ evolves like [1]

$$
\begin{aligned}
\hat{Q}(\tau)= & \sqrt{\frac{\hbar}{2 \Omega_{r} m_{0}}}\left[q^{a}(\tau) \hat{a}+q^{a *}(\tau) \hat{a}^{\dagger}\right] \\
& +\int \frac{d^{3} k}{(2 \pi)^{3}} \sqrt{\frac{\hbar}{2 \omega}}\left[q^{(+)}(\tau, \mathbf{k}) \hat{b}_{\mathbf{k}}+q^{(-)}(\tau, \mathbf{k}) \hat{b}_{\mathbf{k}}^{\dagger}\right],
\end{aligned}
$$

where $q^{a}$ and $q^{( \pm)}$are c-number functions, $\hat{a}, \hat{a}^{\dagger}$ are the lowering and the raising operators of the free detector, and $\hat{b}_{\mathbf{k}}, \hat{b}_{\mathbf{k}}^{\dagger}$ are the annihilation and creation operators of the free field. When sandwiched by the factorized initial state (2) , the two-point functions of the detector and those of the field split into two parts, e.g.,

$$
\left\langle Q(\tau) Q\left(\tau^{\prime}\right)\right\rangle=\langle q \mid q\rangle\left\langle Q(\tau) Q\left(\tau^{\prime}\right)\right\rangle_{\mathrm{v}}+\left\langle Q(\tau) Q\left(\tau^{\prime}\right)\right\rangle_{\mathrm{a}}\left\langle 0_{M} \mid 0_{M}\right\rangle
$$

with

$$
\begin{aligned}
& \left\langle Q(\tau) Q\left(\tau^{\prime}\right)\right\rangle_{\mathrm{v}}= \\
& \left\langle 0_{M}\left|\int \frac{d^{3} k}{(2 \pi)^{3}} \sqrt{\frac{\hbar}{2 \omega}} q^{(+)}(\tau, \mathbf{k}) \hat{b}_{\mathbf{k}} \int \frac{d^{3} k^{\prime}}{(2 \pi)^{3}} \sqrt{\frac{\hbar}{2 \omega^{\prime}}} q^{(-)}\left(\tau^{\prime}, \mathbf{k}^{\prime}\right) \hat{b}_{\mathbf{k}^{\prime}}^{\dagger}\right| 0_{M}\right\rangle, \\
& \left\langle Q(\tau) Q\left(\tau^{\prime}\right)\right\rangle_{\mathrm{a}}= \\
& \frac{\hbar}{2 \Omega_{r} m_{0}}\left\langle q\left|\left[q^{a}(\tau) \hat{a}+q^{a *}(\tau) \hat{a}^{\dagger}\right]\left[q^{a}\left(\tau^{\prime}\right) \hat{a}+q^{a *}\left(\tau^{\prime}\right) \hat{a}^{\dagger}\right]\right| q\right\rangle .
\end{aligned}
$$

Here $\langle. .\rangle_{\mathrm{a}}$ depends on the initial state of the detector only, while $\langle. .\rangle_{\mathrm{v}}$ depends on the initial state of the field, namely the Minkowski vacuum. Therefore by studying the correlation functions $\langle. .\rangle_{\mathrm{a}}$ of the detector and those of the field, one can monitor how the information initially in the detector is flowing into the field.

Indeed, from Ref. [1], we learned that $\left\langle Q^{2}\right\rangle_{\mathrm{a}},\left\langle P^{2}\right\rangle_{\mathrm{a}}$ and $\langle P, Q\rangle_{\mathrm{a}}$ all decay after the coupling is switched on, and the information about the initial state of the detector is subsumed into the quantum field (in $\left\langle\Phi(x) \Phi\left(x^{\prime}\right)\right\rangle_{\mathrm{a}}$, etc.) This view is further supported by the the energy conservation law [1]

$$
\dot{E}(\tau)=-2 \gamma m_{0}\left\langle\dot{Q}^{2}(\tau)\right\rangle_{\text {tot }} .
$$

between the internal energy of the detector (left hand side) and the radiated energy of a monopole radiation (right hand side). Here $\gamma \equiv \lambda_{0}^{2} / 8 \pi m_{0}$ and

$$
E(\tau) \equiv\left\langle\frac{1}{2 m_{0}} P^{2}+\frac{1}{2} m_{0} \Omega_{r}^{2} Q^{2}\right\rangle
$$

is the expectation value of the Hamiltonian of the detector, $\left\langle\dot{Q}^{2}(\tau)\right\rangle_{\text {tot }}$ is the sum of $\left\langle\dot{Q}^{2}(\tau)\right\rangle$ and the interfering term involving vacuum fluctuations of the field [1]. From (44) and (77), one sees that the energy in $\left\langle Q^{2}\right\rangle_{\mathrm{a}}$ and $\left\langle P^{2}\right\rangle_{\mathrm{a}}$ will be converted to monopole radiation while the state of the detector at late times is sustained only by the vacuum fluctuations of the field, namely, the reduced density matrix will be described by $\langle. .\rangle_{\mathrm{v}}$ only. 


\section{A. Purity as a measure of quantum coherence and entanglement}

For the consideration of quantum coherence and entanglement, one needs to go beyond single one-point or two-point functions. The quantity which contains all the information relevant to what we want to investigate here is the reduced density matrix (RDM) $\rho^{R}$, that of the detector when the field degrees of freedom are integrated over, and the RDM of the field when that of the detector is integrated over. Quantum information will be extracted from $\operatorname{Tr}\left(\rho^{R}\right)^{n}, n>1$, which can be expressed as combinations of correlation functions.

Suppose a bipartite system, such as our detector-field system, is in a pure state described by the normalized wave function (al) $\psi[\Phi, Q]$. The density matrix for the combined system in $(\Phi, Q)$ representation reads

$$
\rho\left[\Phi, Q ; \Phi^{\prime}, Q^{\prime}\right]=\psi[\Phi, Q] \psi^{*}\left[\Phi^{\prime}, Q^{\prime}\right]
$$

It is always possible to perform a Schmidt decomposition on the quantum state for the combined system so that [46]

$$
\psi[\Phi, Q]=\sum_{j=1}^{N} M_{j} \Psi_{j}[\Phi] \varphi_{j}(Q)
$$

where $\Psi_{j}[\Phi]$ and $\varphi_{j}(Q)$ are orthonormal states of the field and the detector, respectively. $M_{j}$ 's are non-vanishing coefficients, $N$ in total, chosen to be real here. $N$ is at most equal to the smaller of the dimensionalities of the two subsystems. If $N>1, \psi[\Phi, Q]$ is an entangled (non-separable) state.

The RDM of the detector

$$
\rho^{R}\left(Q, Q^{\prime}\right)=\operatorname{Tr}_{\Phi} \rho=\sum_{j=1}^{N} M_{j}^{2} \varphi_{j}(Q) \varphi_{j}^{*}\left(Q^{\prime}\right)
$$

and the RDM of the field

$$
\rho^{R}\left[\Phi, \Phi^{\prime}\right]=\operatorname{Tr}_{Q} \rho=\sum_{j=1}^{N} M_{j}^{2} \Psi_{j}[\Phi] \Psi_{j}^{*}\left[\Phi^{\prime}\right]
$$

share the same eigenvalues $M_{j}^{2}$. Therefore

$$
\operatorname{Tr}_{Q}\left[\rho^{R}\left(Q, Q^{\prime}\right)\right]^{n}=\operatorname{Tr}_{\Phi}\left(\rho^{R}\left[\Phi, \Phi^{\prime}\right]\right)^{n}=\sum_{j=1}^{N} M_{j}^{2 n}
$$

have the same values from both RDMs for all $n=1,2,3, \ldots$. In particular, the purity $(n=2)$ of the detector has the same value as the purity of the field. The von Neumann entropy $\sim-\rho^{R} \log \rho^{R}=-\sum_{j=1}^{N} M_{j}^{2} \log M_{j}^{2}$ of the detector and of the field are also the same. Note that the form of the equality (13) holds for every choice of time slice where the Hamiltonian for the combined system is defined. The values of $\operatorname{Tr}\left(\rho^{R}\right)^{n}$ for different choice of time slice can be different.

Clearly the purity of each sub-system measures the entanglement between them: For $N=1, \psi$ is factorizable and $\mathcal{P} \equiv \operatorname{Tr}_{Q}\left[\rho^{R}\left(Q, Q^{\prime}\right)\right]^{2}=\mathcal{P}_{\Phi} \equiv \operatorname{Tr}_{\Phi}\left(\rho^{R}\left[\Phi, \Phi^{\prime}\right]\right)^{2}=1$, while for $N>1, \psi$ is entangled and $\mathcal{P}=\mathcal{P}_{\Phi}<1$. On the other hand, the purity of a two-level atom is proportional to its polarization, thus providing a measure of quantum coherence in that atom. Indeed, a two-level atom is not fully decohered until it becomes an infinite-temperature thermal state where the purity is in its least value $1 / 2$. A thermal state at finite temperature, though looks classical, is not fully decohered and the purity is always greater than $1 / 2$. (Note that a diagonal RDM can nonetheless possess nonzero off-diagonal elements under a basis transformation.) Here we extend this view to our system and use the value of the purity function as a measure of quantum coherence in the detector and in the field. (Note that "quantum coherence" used here does not refer to the off-diagonal elements of the density matrix, known to some authors as the "coherences" [47].)

The behavior of quantum coherence "flow" is quite different from energy flow. When the coupling is switched on, both the quantum coherence in the detector and the quantum coherence in the field decrease, while the entanglement between them increases. So quantum coherence does not flow from one subsystem to the other; It goes into sustaining the entanglement between the two subsystems [38]. 


\section{B. RDM of the detector initially in the cat state}

As an example, let us consider the detector in a cat state at the initial moment $\tau_{0}$,

$$
\left|q\left(\tau_{0}\right)\right\rangle=\cos \varphi\left|E_{0}\right\rangle+e^{i \delta} \sin \varphi\left|E_{1}\right\rangle,
$$

where $\left|E_{0}\right\rangle$ and $\left|E_{1}\right\rangle$ are the ground state and the first excited state of the free detector, $\varphi$ is the mixing angle and $\delta$ is a constant phase. The RDM of the detector for the initial state (14) reads

$$
\rho^{R}\left(Q, Q^{\prime} ; \tau\right)=\rho_{(00)}^{R} \cos ^{2} \varphi+\rho_{(11)}^{R} \sin ^{2} \varphi+\left(e^{i \delta} \rho_{(10)}^{R}+e^{-i \delta} \rho_{(01)}^{R}\right) \sin \varphi \cos \varphi
$$

where

$$
\rho_{(m n)}^{R}\left(Q, Q^{\prime} ; \tau\right) \equiv \int \mathcal{D} \Phi_{\mathrm{k}} \psi_{m}\left[Q, \Phi_{\mathrm{k}} ; \tau\right] \psi_{n}^{*}\left[Q^{\prime}, \Phi_{\mathrm{k}} ; \tau\right],
$$

with $m, n=0,1$. Here $\psi_{0}$ is the wave functional corresponding to an initial state given by the tensor product of the Minkowski vacuum and the ground state, and $\psi_{1}$, the product with the first excited state of the detector. In Schrödinger representation, one has

$$
\begin{aligned}
& \rho^{R}\left(Q, Q^{\prime} ; \tau\right)=\sqrt{\frac{G^{11}+G^{22}+2 G^{12}}{\pi}} e^{-G^{i j} Q_{i} Q_{j}} \times \\
& \quad\left\{\cos ^{2} \varphi+\sin ^{2} \varphi\left(C+A^{i j} Q_{i} Q_{j}\right)+\right. \\
& \left.\quad \sin \varphi \cos \varphi\left[\left(e^{i \delta} B^{1}+e^{-i \delta} B^{2 *}\right) Q+\left(e^{-i \delta} B^{1 *}+e^{i \delta} B^{2}\right) Q^{\prime}\right]\right\},
\end{aligned}
$$

where $i, j=1,2, Q_{i}=\left(Q, Q^{\prime}\right)$. The coefficients $C, A^{i j}, B^{j}$, and $G^{i j}$ could be expressed in terms of the correlation functions of the detector by looking at

$$
\begin{aligned}
\left\langle Q^{2}\right\rangle_{m n} & =\operatorname{Tr}\left[Q^{2} \rho_{(m n)}^{R}\right], \\
\langle P, Q\rangle_{m n} & \equiv \frac{1}{2}\langle(P Q+Q P)\rangle_{m n}=\frac{1}{2 i} \operatorname{Tr}\left[Q\left(\partial_{Q}-\partial_{Q^{\prime}}\right) \rho_{(m n)}^{R}\right], \\
\left\langle P^{2}\right\rangle_{m n} & =-\frac{1}{4} \operatorname{Tr}\left[\left(\partial_{Q}^{2}+\partial_{Q^{\prime}}^{2}-2 \partial_{Q} \partial_{Q^{\prime}}\right) \rho_{(m n)}^{R}\right],
\end{aligned}
$$

and so on, where $\langle. .\rangle_{m n} \equiv\left\langle E_{m}, 0_{M}|..| E_{n}, 0_{M}\right\rangle$. Explicit expressions of the two-point functions needed in this paper have been listed in Appendix A of Ref. [2].

The elements of $G^{i j}$ are still the same as those in [1, 2] for the detector initially in the ground state. Expressed in terms of the two-point functions, they are given by

$$
\begin{aligned}
& G^{11}+G^{22}+2 G^{12}=\frac{1}{2\left\langle Q^{2}\right\rangle_{00}} \\
& G^{11}+G^{22}-2 G^{12}=\frac{2}{\hbar^{2}\left\langle Q^{2}\right\rangle_{00}}\left[\left\langle P^{2}\right\rangle_{00}\left\langle Q^{2}\right\rangle_{00}-\left(\langle P, Q\rangle_{00}\right)^{2}\right] \\
& G^{11}-G^{22}=-\frac{i\langle P, Q\rangle_{00}}{\hbar\left\langle Q^{2}\right\rangle_{00}}
\end{aligned}
$$

In writing (17) we have chosen a normalization such that

$$
C=\left\langle Q^{2}\right\rangle_{\mathrm{v}} /\left\langle Q^{2}\right\rangle_{00}
$$

which is zero at the initial moment $\tau=\tau_{0}$ and unity at late times. From (44), (5) and (6), we have $\langle. .\rangle_{00}=$ $\langle. .\rangle_{\mathrm{a}_{0}}+\langle. .\rangle_{\mathrm{v}}$ and $\langle. .\rangle_{11}=3\langle. .\rangle_{\mathrm{a}_{0}}+\langle. .\rangle_{\mathrm{v}}$, where $\langle. .\rangle_{\mathrm{a}_{0}} \equiv\left\langle E_{0}|..| E_{0}\right\rangle$ are the same as those $\langle. .\rangle_{\mathrm{a}}$ in [1, 2]. Then the coefficients $A^{i j}$ in (17) are obtained by solving

$$
\begin{aligned}
& A^{11}+A^{22}+2 A^{12}=\frac{\left\langle Q^{2}\right\rangle_{\mathrm{a}_{0}}}{\left(\left\langle Q^{2}\right\rangle_{00}\right)^{2}}, \\
& A^{11}+A^{22}-2 A^{12}=\frac{4}{\hbar^{2}}\left\{-\left\langle P^{2}\right\rangle_{\mathrm{a}_{0}}+\right. \\
& \left.\quad \frac{\langle P, Q\rangle_{00}}{\left(\left\langle Q^{2}\right\rangle_{00}\right)^{2}}\left[2\langle P, Q\rangle_{\mathrm{a}_{0}}\left\langle Q^{2}\right\rangle_{00}-\left\langle Q^{2}\right\rangle_{\mathrm{a}_{0}}\langle P, Q\rangle_{00}\right]\right\}, \\
& A^{11}-A^{22}=-\frac{2 i}{\hbar\left(\left\langle Q^{2}\right\rangle_{00}\right)^{2}}\left[\langle P, Q\rangle_{\mathrm{v}}\left\langle Q^{2}\right\rangle_{\mathrm{a}_{0}}-\langle P, Q\rangle_{\mathrm{a}_{0}}\left\langle Q^{2}\right\rangle_{\mathrm{v}}\right] .
\end{aligned}
$$


It is known that $\langle. .\rangle_{\mathrm{a}_{0}} \propto e^{-2 \gamma \eta}$ where $\eta \equiv \tau-\tau_{0}[1,2]$, so all $A^{i j}$ vanish at late times.

The coefficients $B^{i}$ can be easily found in a similar way:

$$
\begin{aligned}
B^{1}+B^{2} & =\frac{\langle Q\rangle_{10}}{\left\langle Q^{2}\right\rangle_{00}} \\
B^{1}-B^{2} & =\frac{2 i}{\hbar}\left(\langle P\rangle_{10}-\frac{\langle P, Q\rangle_{00}}{\left\langle Q^{2}\right\rangle_{00}}\langle Q\rangle_{10}\right) .
\end{aligned}
$$

In the Heisenberg picture, one has

$$
\begin{aligned}
& \langle Q\rangle_{10} \equiv\left\langle\left. 0_{M}\right|_{\Phi}\left\langle\left. E_{1}\right|_{Q} \hat{Q}(\tau) \mid E_{0}\right\rangle_{Q} \mid 0_{M}\right\rangle_{\Phi}=\sqrt{\frac{\hbar}{2 \Omega_{r} m_{0}}} q^{\mathrm{a} *}(\tau), \\
& \langle P\rangle_{10}=m_{0}\langle\dot{Q}\rangle_{10}=\sqrt{\frac{\hbar m_{0}}{2 \Omega_{r}}} \dot{q}^{\mathrm{a} *}(\tau),
\end{aligned}
$$

where, as found in Ref. [1],

$$
q^{a}(\eta)=\frac{1}{2} \theta(\eta) e^{-\gamma \eta}\left[\left(1-\frac{\Omega_{r}+i \gamma}{\Omega}\right) e^{i \Omega \eta}+\left(1+\frac{\Omega_{r}+i \gamma}{\Omega}\right) e^{-i \Omega \eta}\right],
$$

where $\Omega_{r}$ is the renormalized natural frequency of the detector and $\Omega \equiv \sqrt{\Omega_{r}^{2}-\gamma^{2}}$. Thus both $\langle Q\rangle_{10}$ and $\langle P\rangle_{10}$ are proportional to $e^{-\gamma \eta}$ and both $\rho_{(10)}^{R}$ and $\rho_{(01)}^{R}$ vanish at late times, when

$$
\left.\rho^{R}\right|_{\gamma \eta \gg 1}=\left.\rho_{(00)}^{R}\right|_{\gamma \eta \gg 1}
$$

for all choices of $\delta$ and $\varphi$ for initial states.

\section{ENTANGLEMENT BETWEEN THE DETECTOR AND THE FIELD}

\section{A. View from the detector}

From (17), the purity of the detector for the initial state (14) is given by

$$
\begin{aligned}
& \mathcal{P}(\tau)=\frac{\hbar}{2 \mathcal{U}}\left(\cos ^{2} \varphi+\frac{\left\langle Q^{2}\right\rangle_{\mathrm{v}}}{\left\langle Q^{2}\right\rangle_{00}} \sin ^{2} \varphi\right)^{2} \\
+ & \frac{\hbar^{3}\left\langle Q^{2}\right\rangle_{00}^{2}}{4 \mathcal{U}^{3}} \sin ^{2} \varphi\left[\left(\cos ^{2} \varphi+\frac{\left\langle Q^{2}\right\rangle_{\mathrm{v}}}{\left\langle Q^{2}\right\rangle_{00}} \sin ^{2} \varphi\right)\left(2 \operatorname{Tr} A \operatorname{Tr} G-8 A^{12} G^{12}\right)\right. \\
& \left.+2 \cos ^{2} \varphi\left(\left|B_{\delta}\right|^{2} \operatorname{Tr} A-2 G^{12} \operatorname{Re}\left(B_{\delta}\right)^{2}\right)\right] \\
+ & \frac{\hbar^{5}\left\langle Q^{2}\right\rangle_{00}^{4}}{8 \mathcal{U}^{5}} \sin ^{4} \varphi\left[6 A^{11} A^{22}(\operatorname{Tr} G)^{2}-24 A^{12} G^{12} \operatorname{Tr} A \operatorname{Tr} G\right. \\
& \left.+\left(\left(A^{11}\right)^{2}+\left(A^{22}\right)^{2}+4\left(A^{12}\right)^{2}\right)\left((\operatorname{Tr} G)^{2}+8\left(G^{12}\right)^{2}\right)\right],
\end{aligned}
$$

where $\operatorname{Tr} A=A^{11}+A^{22}, \operatorname{Tr} G=G^{11}+G^{22}, B_{\delta} \equiv e^{i \delta} B^{1}+e^{-i \delta} B^{2 *}$ and $\mathcal{U}$ is defined in (B2). As shown in Section IIA the purity of the field at the moment $t=a^{-1} \sinh a \tau$ has the same value as (34). At late times $\left(\eta \equiv \tau-\tau_{0} \gg 1 / \gamma\right)$, as the coefficients $A^{i j}$ and $B^{j}$ die out and the RDM is becoming (33), the purity goes to [See (B1)],

$$
\begin{aligned}
\left.\mathcal{P}\right|_{\gamma \eta \gg 1}=\left.\frac{\hbar}{2 \mathcal{U}}\right|_{\gamma \eta \gg 1} \\
=\pi \Omega\left\{\operatorname{Re}\left[\frac{i a}{\gamma+i \Omega}-2 i \psi_{\gamma+i \Omega}\right]\right\}^{-1 / 2} \times \\
\quad\left\{\left(\operatorname{Re}\left\{(\Omega-i \gamma)^{2}\left[\frac{i a}{\gamma+i \Omega}-2 i \psi_{\gamma+i \Omega}\right]\right\}+4 \gamma \Omega\left[\Lambda_{1}-\ln \frac{a}{\Omega}\right]\right)\right\}^{-1 / 2}
\end{aligned}
$$



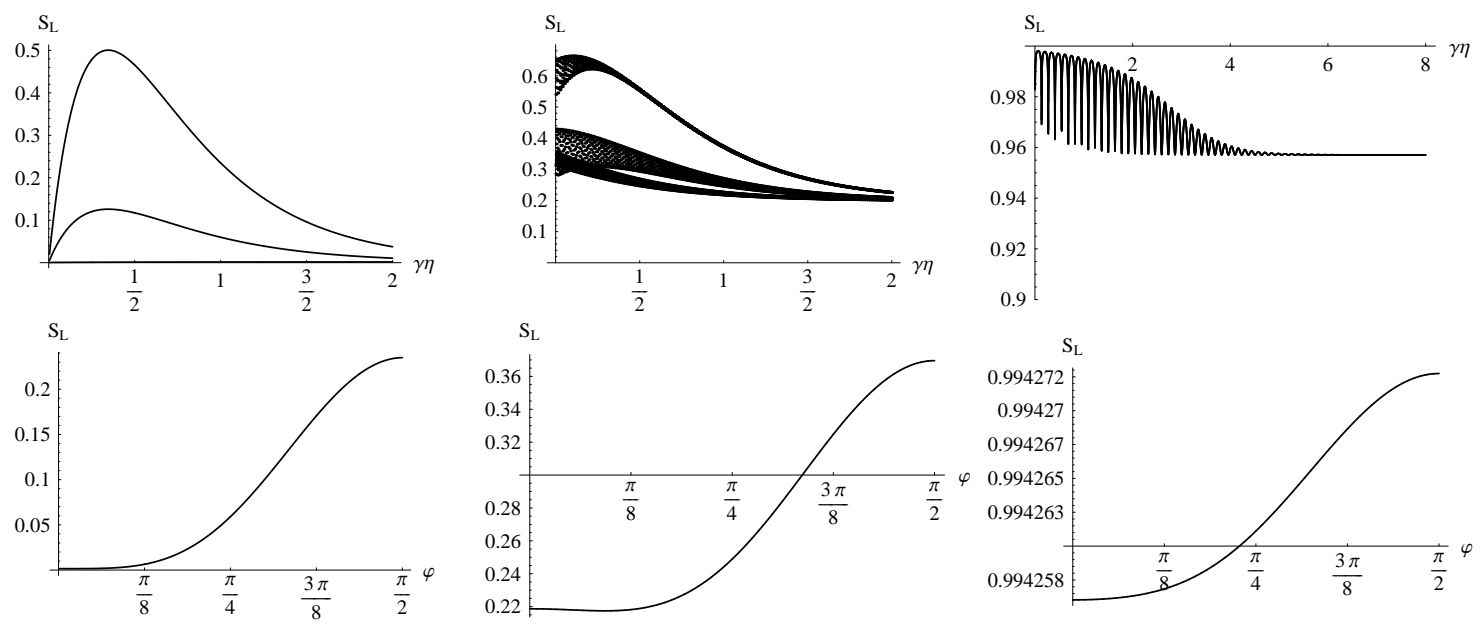

FIG. 1: Linear entropies $S_{L}=1-\mathcal{P}$ of (34) with $\Omega=2.3, a=2, m_{0}=\hbar=1, \Lambda_{1}=\Lambda_{0}=10000, \delta=0$. From left to right, $\gamma=10^{-7}, 10^{-4}, 0.1$, respectively. (Upper row): Evolution of $S_{L}$ in detector's proper time $\eta \equiv \tau-\tau_{0}$ with the initial moment $\tau_{0}$. The three curves from top to bottom in each plot (the bottom one in the left plot is very close to the $\gamma \eta$ axis, while the three curves are indistinguishable in the right plot) have $\varphi=\pi / 2, \pi / 4,0$, respectively. (Lower row): $S_{L}$ against the mixing angle $\varphi \in(0, \pi / 2)$ at the moment $\eta=1 / \gamma$.

whose value is determined by the vacuum fluctuations, for all initial states of the detector [1]. Here $\psi_{s} \equiv \psi\left(1+a^{-1} s\right)$ is the digamma function.

In Sec. II we stated that for a bipartite system in a pure state the purity function measures quantum coherence in each subsystem and the entanglement between two subsystems: a lower purity means lesser quantum coherence in the detector or the field and stronger entanglement between the detector and the field. To show this one may define the linear entropy in terms of the purity as

$$
S_{L} \equiv 1-\mathcal{P}
$$

Now the value of $S_{L}$ is zero for a detector in a pure state and is positive for a detector in a mixed state. By definition the linear entropy seen by the detector will be equal to the linear entropy seen by the field. Also, from Sec. ЩA it is easy to see that the greater the von Neumann entropy of the detector, the greater $S_{L}$. More precisely, for a pure state of a bipartite system, $S_{L}$ is a Schur concave function of the Schmidt vector $M_{j}^{2}$ in (11), and $S_{L}$ is invariant under unitary operations and decrease, on average, under local operations and classical communications. Thus $S_{L}$ is a entanglement monotone [48, 49], which can serve as a measure of entanglement between the detector and the field, just as good as the von Neumann entropy.

The linear entropies for different cases are illustrated in FIG. 1. We find that, during the evolution, the UD detector with the first excited state $\left|E_{1}\right\rangle$ as the initial state $(\varphi=\pi / 2)$ always has a larger linear entropy, hence a stronger entanglement with the field, than those detectors initially prepared in cat states or the ground state $(0 \leq \varphi<\pi / 2)$ of the free detector.

\section{B. recoherence in ultraweak coupling regime}

In the ultraweak coupling regime $\left(\gamma \Lambda_{1} \ll a, \Omega\right)[2]$,

$$
\begin{aligned}
\left\langle Q^{2}\right\rangle_{\mathrm{v}} & \approx \frac{\hbar}{2 \Omega m_{0}} \operatorname{coth} \frac{\pi \Omega}{a}\left(1-e^{-2 \gamma \eta}\right), \\
\left\langle Q^{2}\right\rangle_{\mathrm{a}_{0}} & \approx \frac{\hbar}{2 \Omega m_{0}} e^{-2 \gamma \eta},
\end{aligned}
$$

$\left\langle P^{2}\right\rangle_{\mathrm{v}, \mathrm{a}_{0}} \approx m_{0}^{2} \Omega^{2}\left\langle Q^{2}\right\rangle_{\mathrm{v}, \mathrm{a}_{0}}$ and $\langle P, Q\rangle_{\mathrm{v}, \mathrm{a}_{0}}$ are negligible, thus

$$
\mathcal{U} \approx \frac{\hbar}{2}\left[e^{-2 \gamma \eta}+\left(1-e^{-2 \gamma \eta}\right) \operatorname{coth} \frac{\pi \Omega}{a}\right],
$$


and

$$
\begin{aligned}
\mathcal{P} \approx & \left(\frac{\hbar}{2 \mathcal{U}}\right)^{3} e^{-4 \gamma \eta}\left\{\left[\cos ^{2} \varphi+\left(e^{2 \gamma \eta}-1\right) \operatorname{coth} \frac{\pi \Omega}{a}\right]^{2}\right. \\
& \left.+2 \cos ^{2} \varphi \sin ^{2} \varphi\left[1+\left(e^{2 \gamma \eta}-1\right) \operatorname{coth} \frac{\pi \Omega}{a}\right]+\sin ^{4} \varphi\right\}
\end{aligned}
$$

For $a / \Omega$ sufficiently small, one has

$$
\mathcal{P} \approx 1+2 e^{-2 \gamma \eta}\left(e^{-2 \gamma \eta}-1\right) \sin ^{4} \varphi .
$$

One can see that in this regime $\mathcal{P}$ is very close to unity at late times, when each subsystem re-gains almost all quantum coherence and turns into a nearly pure state.

Indeed, observing the upper-left plot of FIG. 1, the linear entropy $S_{L}$ in the detector increases from zero right after the coupling is switched on, reaches a maximum $(1 / 2) \sin ^{4} \varphi$ at $\eta \approx \ln 2 / 2 \gamma$, then decays to a small common value that detectors with all other initial states will asymptopte to. This decay of the degree of entanglement or the restoration of the degree of quantum coherence is known as "recoherence" [34]. From (35) one sees that the late-time recoherence manifests only in the ultraweak coupling regime with sufficiently low acceleration (temperature), where the late-time $\mathrm{RDM}$ of the detector looks very close to the density matrix of the ground state of a free detector. Thus the recoherence here characterizes the process of relaxation or spontaneous emission by which the detector initially in excited state will finally fall into a steady state which is very close to the ground state of the free detector. Nevertheless, full recoherence is impossible once the coupling is on, since the late-time linear entropy

$$
\begin{aligned}
&\left.S_{L}\right|_{\gamma \eta \rightarrow \infty} \approx 1-\tanh \frac{\pi \Omega}{a}+\gamma \frac{2 \tanh ^{2} \frac{\pi \Omega}{a}}{\pi \Omega} \times \\
& {\left[\Lambda_{1}+\ln \frac{\Omega}{a}-\operatorname{Re}\left[\psi\left(\frac{i \Omega}{a}\right)+\frac{i \Omega}{a} \psi^{(1)}\left(\frac{i \Omega}{a}\right)\right]\right]+O\left(\gamma^{2}\right) } \\
& \stackrel{a \ll \Omega}{\longrightarrow} \frac{2 \gamma}{\pi \Omega}\left(\Lambda_{1}-1\right)+O\left(\gamma^{2}\right),
\end{aligned}
$$

$\left(\psi^{(1)}(x) \equiv d \psi(x) / d x\right)$ remains nonzero for any positive $\gamma$, even when $a \rightarrow 0$.

Eq. (37) yields

$$
\left\langle Q^{2}\right\rangle_{11} \approx \frac{\hbar}{2 \Omega m_{0}}\left(1+2 e^{-2 \gamma \eta}\right)
$$

and $\left\langle P^{2}\right\rangle_{11} \approx m_{0}^{2} \Omega^{2}\left\langle Q^{2}\right\rangle_{11}$ in the ultraweak coupling limit. When $\varphi=\pi / 2$ and $\eta=\ln 2 / 2 \gamma,\left.\left\langle Q^{2}\right\rangle_{11}\right|_{\eta=\ln 2 / 2 \gamma}=$ $\left(\left\langle Q^{2}(0)\right\rangle_{00}+\left\langle Q^{2}(0)\right\rangle_{11}\right) / 2$ and $\left.\left\langle P^{2}\right\rangle_{11}\right|_{\eta=\ln 2 / 2 \gamma}=\left(\left\langle P^{2}(0)\right\rangle_{00}+\left\langle P^{2}(0)\right\rangle_{11}\right) / 2$. It may seem that the intermediate state of the detector during the transition is a cat state which is a superposition of the ground state and the first excited state of the free detector, but this is not true. Recall that the Einstein A coefficient describing the spontaneous emission of an atom in a thermal bath of photons pertains to transition probability rather than amplitude. Large $S_{L}$ in transient indicates that the intermediate state during the spontaneous emission is a mixed state, in contrast to the zero $S_{L}$ for a pure cat state (at the initial moment of the middle curve in the upper-left plot of FIG. 10). The value of $\langle. .\rangle_{11}$ in transient is mainly an ensemble (probabilistic) average of the population in the ground state and the population in the first excited state. Indeed, transformed to the representation of energy eigenstates of the free detector, the RDM of the detector becomes

$$
\begin{aligned}
& \rho_{0,0}^{R} \approx \cos ^{2} \varphi+\left(1-e^{-2 \gamma \eta}\right) \sin ^{2} \varphi, \\
& \rho_{1,1}^{R} \approx e^{-2 \gamma \eta} \sin ^{2} \varphi \\
& \rho_{0,1}^{R}=\left(\rho_{1,0}^{R}\right)^{*} \approx e^{-(\gamma-i \Omega) \eta+i \delta} \cos \varphi \sin \varphi,
\end{aligned}
$$

with other elements negligible. When $\varphi=\pi / 2$, it behaves like the population of the first excited state $\rho_{1,1}^{R}$ decaying to the ground state directly while $\rho_{0,1}^{R}$ and $\rho_{1,0}^{R}$ are always negligible.

\section{Beyond the ultraweak coupling regime}

As the coupling increases, $\Lambda_{0}$ and $\Lambda_{1}$ terms [1, 2] take over (see upper-middle plot of FIG. 1) and dominate (upperright, FIG. (1). The emerging initial oscillations of $S_{L}$ is coming from the $\Lambda_{0}$ term, which corresponds to the time-scale 


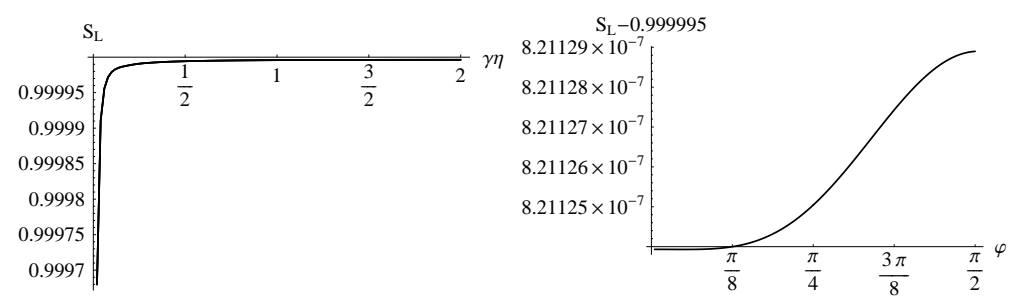

FIG. 2: $S_{L}$ in ultrahigh acceleration limit: $a=2 \times 10^{6}, \gamma=0.1$, other parameters have the same values as those in FIG. 1 As indicated in the right plot, the curves with different $\varphi$ are not distinguishable in the left and middle plots.

of the switching and would be tamed by turning on the coupling smoothly, while the large late-time $S_{L}$ (or the small $\mathcal{P})$ is due to the $\Lambda_{1}$ term, which corresponds to the time-resolution of this theory.

When $\gamma \Lambda_{1}$ is comparable with or greater than $a$ and $\Omega$, the system is in the non-Markovian regime and the purity is always small, which implies that the detector experiences strong decoherence associated with strong entanglement between the detector and the field. The behavior of the detector is dominated by the physical cut-offs $\left(\Lambda_{0}\right.$ and $\left.\Lambda_{1}\right)$ and the differences between initial states of the detector can be negligible (see upper-right plot of FIG. 1.) For example, when $\varphi=\pi / 2$, just like the case with the detector initially in its ground state, the initial distribution of the RDM in energy-eigenstate representation $\rho_{m, n}^{R}$ peaked at the element $\rho_{1,1}^{R}$ would, upon the switch-on of the coupling, collapse rapidly into a distribution widely spread over the whole density matrix, for which the energy eigenstates of the free detector cannot form a good basis because the off-diagonal terms of the RDM do not vanish even in steady state [2]. The late-time linear entropy

$$
\left.S_{L}\right|_{\gamma \eta \gg 1} \approx 1-\frac{\pi / 2}{\sqrt{\frac{2}{\Omega} \gamma \Lambda_{1} \operatorname{Re}\left[\frac{i a}{\gamma+i \Omega}-2 i \psi_{\gamma+i \Omega}\right]}}+O\left(\Lambda_{1}^{-3 / 2}\right)
$$

is still very close to unity. Hence there is no late-time recoherence in this regime, where the quantum state of the combined system is anything but a direct product of the state of the detector and that of the field.

A large linear entropy at late times also shows up at the ultrahigh acceleration (or Unruh temperature) limit $\left(a \gg \gamma \Lambda_{1}, \Omega\right)$

$$
\left.S_{L}\right|_{\gamma \eta \gg 1} \approx 1-\frac{\pi \Omega_{r}}{2 a}+O\left(a^{-2}\right)
$$

(see FIG. 2). When the coupling is weak enough, the energy eigenstates of the free detector can still form a good basis and the RDM of the detector is approximately a thermal state in energy-eigenstate representation: all off-diagonal elements are negligible. Note that the ultrahigh temperature limit is still in a Markovian regime, so we see that strong entanglement does not imply a non-Markovian process. Although the difference between the initial and the final state looks large in this limit, the detector would feel that the state of the field is not heavily altered by the detector [2]. This would become clear from the viewpoint of the field which we now turn to.

\section{View from the field}

Eq. (39) implies that

$$
\left.\frac{d S_{L}}{d \tau}\right|_{\tau \rightarrow \tau_{0}} \approx \gamma\left[\frac{1}{2} \operatorname{coth} \frac{\pi \Omega}{a}(7-4 \cos 2 \varphi+\cos 4 \varphi)-2\right],
$$

in the ultraweak coupling limit. From which one sees that the larger the proper acceleration $a$ is, the steeper is the initial rising rate for $S_{L}$ in the proper time of the detector, and the larger is the linear entropy at fixed $\tau$ in transient. In other words, for ultraweak interactions, entanglement grows faster in transient for larger $a$ measured by the clock of the detector. However, the detector with a larger $a$ has a more pronounced time dilation for the Minkowski observer (see FIG. 3), so the linear entropy for a larger $a$ does not have to grow faster in Minkowski time $t$. In fact, in FIG. 4. we see that for sufficiently small $a, S_{L}$ at fixed Minkowski time can decrease as $a$ increases.

From the viewpoint of the field, a uniformly accelerated detector with large $a$ has obvious changes in time only around $t=0$ because significant time-dilation will be seen by the field once $|t|$ is large enough. Therefore from the viewpoint of the field most of the time the detector actually looks frozen and the radiated power per unit Minkowski time is small [1], so the field seems not strongly affected by the detector. 


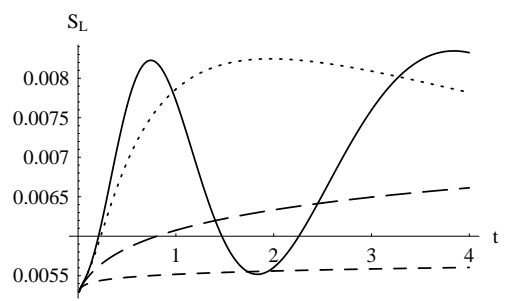

FIG. 3: Evolution of $S_{L}$ in Minkowski time $t$ after the coupling is switched on at $t=0$. Here $\gamma=.0001, \Omega=2.3, \Lambda_{0}=\Lambda_{1}=100$, $\varphi=\pi / 4, a=1,4,16,64$ for the solid, dotted, long-dashed and short-dashed curves, respectively. One can see that the larger $a$, the more pronounced time-dilation for $S_{L}$ in Minkowski time $t$. Note that this plot is still in the weak coupling regime.

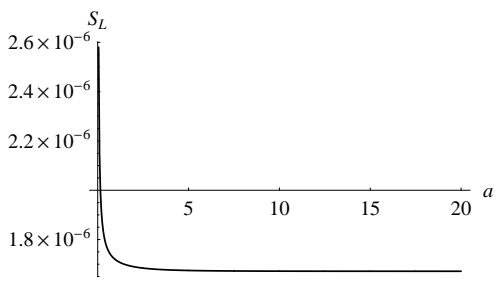

FIG. 4: $S_{L}$ against $a$ at fixed Minkowski time $t=10^{6}$. The coupling is switched on at $t_{0}=0$. Here $\gamma=10^{-9}, \Omega=2.3$, $\Lambda_{0}=\Lambda_{1}=1000, \varphi=\pi / 2$, other parameters are the same as those in FIG. 1 .

\section{SUMMARY AND DISCUSSION}

\section{A. Summary}

Applying the non-perturbative results obtained in [1, 2], we analyze the evolution of quantum coherence and entanglement in a uniformly accelerated detector - quantum field system. By studying the evolution of a UD detector initially in a superposition of energy eigenstates of the free detector and in contact with a massless scalar field in the Minkowski vacuum, we demonstrate that only in the very restrictive regime of ultraweak coupling, while the detector loses quantum coherence during spontaneous emission when the detector makes transit from its initial state to the final state very close to its free ground state, an imperfect recoherence will occur eventually. This can be considered as qualitatively consistent with the claims of [34]. In the much broader range where the coupling is not ultraweak, our explicit calculation shows the detector has a very low purity at late times, thus quantum coherence never returns to the detector and quantum entanglement between the detector and the field is always very strong. We now discuss a few questions and issues brought up in this investigation.

\section{B. Is there transient recoherence during spontaneous emission?}

We have done a similar calculation for the detector initially in the second excited state. In the ultraweak coupling limit with sufficiently small $a / \Omega$, the evolution of purity can be approximated by

$$
\mathcal{P} \approx 1-4 e^{-2 \gamma \eta}\left(1-e^{-2 \gamma \eta}\right)+6\left[e^{-2 \gamma \eta}\left(1-e^{-2 \gamma \eta}\right)\right]^{2},
$$

which behaves quite similar to the case when the detector is initially in the first excited state $(\varphi=\pi / 2$ in (40) and the top curve in the upper-left plot of FIG.1.) There exists only one extremum for (49): Again when $\eta \approx \ln 2 / 2 \gamma, \mathcal{P}$ has the minimal value $3 / 8$. Thus there is no "transient recoherence" or intermediate pure state during the spontaneous emission process for the UD detector-field system. While $\left.\left\langle Q^{2}\right\rangle_{22}\right|_{\eta=\ln 2 / 2 \gamma}$ has the value of $\left\langle Q^{2}(0)\right\rangle_{11}$, which makes the detector look like it is in its first excited state, the detector is actually in a mixture of the second, the first and the ground state since $S_{L}$ is large then. 


\section{Superposition and coherence: quantum vs classical}

The oscillating mean field emitted by the detector is a manifestation of quantum superposition of energy eigenstates inside the detector, but the converse is not true. An example is that if the initial state of the detector is a superposition of its ground state and the second excited state, the mean field emitted by the detector is always zero.

One may ask whether the mean field carries "quantum information" rather than a classical one. The expectation values of $Q$ and $P$ and the mean field actually enter into the energy conservation law (7) between the internal energy of the detector and the radiated energy of a monopole radiation emitted by the detector. As far as the energy balance is concerned there is no essential difference between the mean field and the classical field. From this view the mean field carries no quantum information.

To determine the initial state of the detector from the evolving quantum field, an observer without any a priori knowledge about the set-up has to find out the correlation functions to all orders, even though in the problem at hand only the one-point (mean field) and two-point functions of the detector and those of the field are sufficient as they contain the full information about the combined system. This is because the UD detector theory is linear and so all higher-order correlation functions can be written as combinations of these lower-order functions.

\section{Information in a mixed state vs. No information in a thermal state}

As long as the coupling between the detector and the field is on, the detector and the field are separately in a mixed state of its own, while the combined system remains in a pure state. The mixed state of the detector carries information of the initial state until $\left\langle Q^{2}\right\rangle_{a},\left\langle P^{2}\right\rangle_{a}$ and $\langle P, Q\rangle_{a}$ all decay away and the detector reaches a steady state. At late times, while the energy eigenstates of the free detector cannot form a good basis, the RDM of the detector can be diagonalized to a Boltzmann distribution from which one can read off the same effective temperature as those reported in [2]. In this sense the final mixed state of the detector is a thermal state containing no initial information. This is true for both inertial and uniformly accelerating detectors, the former case might be a surprise.

\section{E. Detector near a black hole event horizon}

The case of a uniformly accelerated detector in Minkowski space is analogous to the case of a detector sitting outside a Schwarzschild black hole at a fixed radial distance. In [1] we have shown that the information about the initial state of the detector will be carried in (quantum) radiation emitted and propagated to the future null infinity $\mathcal{I}_{+}$of Minkowski space, and all the radiation containing those information eventually cross the event horizon of the detector. In the black hole case, by contrast, a quick estimate indicates that not all, but about one half, of the radiation emitted from the detector will enter the event horizon of the black hole, while the remaining radiation goes outwards to $\mathcal{I}_{+}$. The exact ratio between the inward and outward radiations depends on the gray body factor of the black hole and could be obtained only by detailed calculations.

Now consider the situation that, besides this fixed detector, there is another detector carrying some information and falling into the black hole. One may ask how information might flow from the free-falling detector to the fixed one, and how the correlations between these two detectors evolve. It is also interesting to look into the way how the quantum field aids in the propagation of information and the evolution of those correlations. We will answer these questions in a forthcoming paper.

\section{F. Black hole information and end state}

Another avenue to connect with the black hole problem is to treat the detector itself as an analog of the black hole. This follows Bekenstein's observation that the black hole behaves like an ensemble of quantum mechanical atoms $[50,51,52,53]$, whose spontaneous emissions correspond to Hawking radiation, and the energy level of the atom is analogous to the area level of the black hole. When they are fed with field quanta, black holes tend to absorb more than emit energy. Indeed, Bekenstein and Meisels showed that for black holes the Einstein B coefficient for stimulated absorption is greater than the coefficient for stimulated emission [51].

Let us assume that the theory for black hole atoms does not violate unitarity and there is no information loss, just like our UD detector theory. Then our results for $a=0$ indicate that, if the field is initially in a vacuum state, the information about the black hole will be encoded in its spontaneous emission, namely, its emitted radiation which is not exactly thermal [54]. All initial information in the black hole will eventually go to the field at late times, while the final state of the black hole is sustained by the vacuum fluctuations of the quantum field. This is consistent with the 
"no-hiding theorem" of Braunstein and Pati (with their ancilla as our quantum field) [40]: no information is hidden in the correlations between the field and the black hole. One can further verify this statement by noting that the correlation functions $\langle Q(\tau) \Phi(t(\tau), \mathbf{x})\rangle_{\mathrm{a}}$ for all $\mathbf{x}$ vanish at late times.

When the black hole is radiating, the black hole itself and the field outside the black hole are each in a mixed state. Only in the ultraweak coupling limit can they restore most of their purity at late times. Otherwise, in the more prevalent non-Markovian regime, the area eigenstates of the black hole cannot form a good basis, and the entanglement between the black hole and the field is always large. Nevertheless, in this scenario the quantum state of the combined system is always pure due to the unitarity we assumed.

In our model the difference between the degrees of freedom of the detector and those of the field is put in by hand and never disappears, and we cannot address whether the ground state corresponds to a black hole remnant, an ordinary localized mass, or nothing. However, in Bekenstein's atom analog highly excited eigenstates correspond to large-area black holes. Our results indicate that in the rather general non-Markovian regime, the combined system would evolve to a highly entangled state between the black hole and the field, and the final state of the black hole would be a complicated mixed state distributed widely from the ground state to the highly excited area-eigenstates. So at late times in the broad ranged non-Markovian regime the black hole could end up as a remnant with a large mean area with all its initial information already leaked out and dispersed into the quantum field.

Acknowledgement SYL wishes to thank Zheng-Yao Su for helpful discussions. BLH appreciates the hospitality of Stephen Adler while visiting the Institute for Advanced Study, Princeton in Spring '07. This work is supported in part by an NSF Grant PHY-0601550.

\section{APPENDIX A: RDM OF THE QUANTUM FIELD INITIALLY IN THE MINKOWSKI VACUUM}

The RDM of the field for the initial condition (14) is similar in form as (17):

$$
\begin{gathered}
\rho^{R}\left(\Phi, \Phi^{\prime} ; t\right)=\frac{e^{-\gamma_{i j}^{\mathrm{xy}}(t) \Phi_{\mathrm{x}}^{i} \Phi_{\mathrm{y}}^{j}}}{\sqrt{\operatorname{det}\left(2 \pi D_{\mathrm{xy}}\right)}}\left\{\cos ^{2} \varphi+\sin ^{2} \varphi\left(\chi(t)+\alpha_{i j}^{\mathrm{xy}}(t) \Phi_{\mathrm{x}}^{i} \Phi_{\mathrm{y}}^{j}\right)+\right. \\
\left.\sin \varphi \cos \varphi\left[\left(e^{i \delta} \beta_{1}^{\mathrm{x}}+e^{-i \delta} \beta_{2}^{\mathrm{x} *}\right) \Phi_{\mathrm{x}}+\left(e^{-i \delta} \beta_{1}^{\mathrm{x} *}+e^{i \delta} \beta_{2}^{\mathrm{x}}\right) \Phi_{\mathrm{x}}^{\prime}\right]\right\},
\end{gathered}
$$

where $\Phi_{\mathrm{x}}^{j}=\left(\Phi_{\mathrm{x}}, \Phi_{\mathrm{x}}^{\prime}\right), j=1,2$ and

$$
D_{\mathrm{xy}}(t) \equiv\left\langle\Phi_{\mathrm{x}}(t), \Phi_{\mathrm{y}}(t)\right\rangle_{00}
$$

is symmetric by definition: $\langle\hat{A}, \hat{B}\rangle \equiv\langle(\hat{A} \hat{B}+\hat{B} \hat{A})\rangle / 2$. Writing the inverse matrix of $D_{\mathrm{xy}}$ as $D^{\mathrm{xy}}$ so that $D_{\mathrm{xy}} D^{\mathrm{yz}}=$ $\delta_{\mathrm{x}}{ }^{\mathrm{z}}=\delta^{3}(\mathrm{x}-\mathrm{z})$, the coefficients in (A1) obey the following relations

$$
\begin{aligned}
& \left(\gamma_{11}+\gamma_{22}+\gamma_{12}+\gamma_{21}\right)^{\mathrm{xy}}=\frac{1}{2} D^{\mathrm{xy}}, \\
& \left(\gamma_{11}-\gamma_{22}+\gamma_{12}-\gamma_{21}\right)^{\mathrm{xy}}=-\frac{i}{\hbar}\left\langle\Pi^{\mathrm{x}}, \Phi_{\mathrm{z}}\right\rangle_{00} D^{z y}, \\
& \left(\gamma_{11}+\gamma_{22}-\gamma_{12}-\gamma_{21}\right)^{\mathrm{xy}}= \\
& \frac{2}{\hbar^{2}}\left[\left\langle\Pi^{\mathrm{x}}, \Pi^{\mathrm{y}}\right\rangle_{00}-\left\langle\Pi^{\mathrm{x}}, \Phi_{\mathrm{z}}\right\rangle_{00} D^{\mathrm{zz}}\left\langle\Pi^{\mathrm{y}}, \Phi_{\mathrm{z}^{\prime}}\right\rangle_{00}\right], \\
& \left(\alpha_{11}+\alpha_{22}+\alpha_{12}+\alpha_{21}\right)^{\mathrm{xy}}=\left\langle\Phi_{\mathrm{z}}, \Phi_{\mathrm{z}^{\prime}}\right\rangle_{\mathrm{a}_{0}} D^{\mathrm{zx}} D^{\mathrm{z}^{\prime} \mathrm{y}}, \\
& \left(\alpha_{11}-\alpha_{22}+\alpha_{12}-\alpha_{21}\right)^{\mathrm{xy}}= \\
& \frac{2 i}{\hbar}\left[\left\langle\Pi^{\mathrm{x}}, \Phi_{\mathrm{z}}\right\rangle_{\mathrm{a}_{0}} D^{\mathrm{zy}}-\left\langle\Pi^{\mathrm{x}}, \Phi_{\mathrm{z}}\right\rangle_{00} D^{\mathrm{zv}}\left\langle\Phi_{\mathrm{v}}, \Phi_{\mathrm{w}}\right\rangle_{\mathrm{a}_{0}} D^{\mathrm{wy}}\right], \\
& \left(\alpha_{11}+\alpha_{22}-\alpha_{12}-\alpha_{21}\right)^{\mathrm{xy}}= \\
& -\frac{4}{\hbar^{2}}\left[\left\langle\Pi^{\mathrm{x}}, \Pi^{\mathrm{y}}\right\rangle_{\mathrm{a}_{0}}+\left\langle\Pi^{\mathrm{x}}, \Phi_{\mathrm{w}}\right\rangle_{00} D^{\mathrm{wz}}\left\langle\Phi_{\mathrm{z}}, \Phi_{\mathrm{z}^{\prime}}\right\rangle_{\mathrm{a}_{0}} D^{\mathrm{z}^{\prime} \mathrm{w}^{\prime}}\left\langle\Pi^{\mathrm{y}}, \Phi_{\mathrm{w}^{\prime}}\right\rangle_{00}\right. \\
& \left.-D^{\mathrm{zz}}\left(\left\langle\Pi^{\mathrm{x}}, \Phi_{\mathrm{z}}\right\rangle_{00}\left\langle\Pi^{\mathrm{y}}, \Phi_{\mathrm{z}^{\prime}}\right\rangle_{\mathrm{a}_{0}}+\left\langle\Pi^{\mathrm{x}}, \Phi_{\mathrm{z}}\right\rangle_{\mathrm{a}_{0}}\left\langle\Pi^{\mathrm{y}}, \Phi_{\mathrm{z}^{\prime}}\right\rangle_{00}\right)\right], \\
& \left(\beta_{1}+\beta_{2}\right)^{\mathrm{x}}=\left\langle\Phi_{\mathrm{z}}\right\rangle_{10} D^{\mathrm{zx}}, \\
& \left(\beta_{1}-\beta_{2}\right)^{\mathrm{x}}=\frac{2 i}{\hbar}\left[\left\langle\Pi^{\mathrm{x}}\right\rangle_{10}-\left\langle\Phi_{\mathrm{z}}\right\rangle_{10}\left\langle\Pi^{\mathrm{x}}, \Phi_{\mathrm{z}^{\prime}}\right\rangle_{00} D^{\mathrm{zz}}\right] .
\end{aligned}
$$


Here $\Pi^{\mathrm{x}}$ is the conjugate momentum of $\Phi_{\mathrm{x}}$, and we choose

$$
\chi=1-\left\langle\Phi_{\mathrm{z}}, \Phi_{\mathrm{z}^{\prime}}\right\rangle_{\mathrm{a}_{0}} D^{\mathrm{zz}} .
$$

Note that $\gamma_{11}^{\mathrm{xy}}, \gamma_{22}^{\mathrm{xy}}, \alpha_{11}^{\mathrm{xy}}$ and $\alpha_{22}^{\mathrm{xy}}$ are symmetric under $(\mathrm{x} \leftrightarrow \mathrm{y})$, so are $\left\langle\Phi_{\mathrm{x}}, \Phi_{\mathrm{y}}\right\rangle_{00},\left\langle\Pi^{\mathrm{x}}, \Pi^{\mathrm{y}}\right\rangle_{00},\left\langle\Phi_{\mathrm{x}}, \Phi_{\mathrm{y}}\right\rangle_{\mathrm{a}_{0}}$ and $\left\langle\Pi^{\mathrm{x}}, \Pi^{\mathrm{y}}\right\rangle_{\mathrm{a}_{0}}$. Thus the total number of degrees of freedom for $\gamma_{i j}^{\mathrm{xy}}$ and $\alpha_{i j}^{\mathrm{xy}}$ are exactly the same as the number for the two-point functions of the field, as enumerated in the above equations (note that (A3), (A5), (A6) and (A8) are symmetric).

\section{APPENDIX B: PURITIES FOR DETECTOR INITIALLY IN THE GROUND STATE}

For a UD detector initially in the ground state, the purity is given by (34) with $\varphi=0$ :

$$
\mathcal{P}=\sqrt{\frac{\left(G^{11}+G^{22}+2 G^{12}\right)^{2}}{\operatorname{det}\left[G^{i j}+\left(G^{i j}\right)^{*}\right]}}=\frac{\hbar / 2}{\mathcal{U}}
$$

where

$$
\mathcal{U} \equiv \sqrt{\left\langle P^{2}\right\rangle_{00}\left\langle Q^{2}\right\rangle_{00}-\left(\langle P, Q\rangle_{00}\right)^{2}}
$$

is the uncertainty function and $\mathcal{U} \geq \hbar / 2$ is the Robertson-Schrödinger uncertainty relation. The physics of the detector with such an initial state has been studied in detail in Ref. 2].

The purity of the field (A1) for the same initial state is

$$
\begin{aligned}
& \left.\mathcal{P}_{\Phi}\right|_{\varphi=0}=\left\{\operatorname { d e t } \left[\frac { 4 } { \hbar ^ { 2 } } \left(\left\langle\Phi_{\mathrm{x}} \Phi_{\mathrm{y}}\right\rangle_{00}\left\langle\Pi^{\mathrm{y}} \Pi^{\mathrm{z}}\right\rangle_{00}-\right.\right.\right. \\
& \left.\left.\left.\left\langle\Phi_{\mathrm{x}} \Phi_{\mathrm{y}}\right\rangle_{00}\left\langle\Pi^{\mathrm{y}}, \Phi_{\mathrm{w}}\right\rangle_{00} D^{\mathrm{ww}^{\prime}}\left\langle\Phi_{\mathrm{w}^{\prime}}, \Pi^{\mathrm{z}}\right\rangle_{00}\right)\right]\right\}^{-1 / 2} .
\end{aligned}
$$

One can compare this with (B1) to see the similarity in appearance. Note that, while (B1) is parametrized in the proper time of the detector $\tau$, the time variable of (B3) is the Minkowski time $t$, and $\mathrm{x}, \mathrm{y}, \mathrm{z}, \mathrm{w}$ in the above expression are defined in the whole Minkowski-time slice rather than in the Rindler R-wedge only since the initial state of the field is the Minkowski vacuum.

Although (B3) looks a bit complicated for an analytic expression, (13) guarantees that (B1) and (B3) have the same value when $t=a^{-1} \sinh a \tau$.

\section{APPENDIX C: COMPARISON WITH ANGLIN ET AL. [34]}

The authors of Ref. [34] considered a model with a harmonic oscillator at rest interacting with a scalar field in $(1+1)$ D. While the appearance of their action looks quite different from (1), in reality their harmonic oscillator is acting like a UD detector initially in contact with an ohmic bath. Thus when focusing on the detector, we can borrow our results in Ref. 2] for UD detector at rest $(a=0)$ to describe the behavior of their harmonic oscillator with a step switching function.

Ref. [34] assumes that the combined system has the initial state:

$$
\left|\psi\left(\tau_{0}\right)\right\rangle=\left(c_{+}|\alpha\rangle+c_{-}|-\alpha\rangle\right) \otimes\left|0_{M}\right\rangle,
$$

where $|\alpha\rangle$ is a coherent state of the detector, which is an eigenstate of the lowering operator $\hat{a}$ :

$$
\hat{a}|\alpha\rangle=\alpha|\alpha\rangle \text {. }
$$

Here the eigenvalue is

$$
\alpha=\sqrt{\frac{m_{0} \Omega_{r}}{2 \hbar}} q_{0}
$$


with the initial mean value $\left\langle\alpha\left|\hat{Q}\left(\tau_{0}\right)\right| \alpha\right\rangle=q_{0}$ of the detector. (Note a transcription oversight in Ref. [1]: $\alpha$ should carry the $\sqrt{m_{0}}$ factor.) The normalization condition is

$$
1=\left\langle\psi\left(\tau_{0}\right) \mid \psi\left(\tau_{0}\right)\right\rangle=\left|c_{+}\right|^{2}+\left|c_{-}\right|^{2}+\left(c_{+}^{*} c_{-}+c_{+} c_{-}^{*}\right) e^{-m_{0} \Omega_{r} q_{0}^{2} / \hbar} .
$$

If we require $\left|c_{+}\right|^{2}+\left|c_{-}\right|^{2}=1$, then $c_{+}^{*} c_{-}+c_{+} c_{-}^{*}$ must vanish; we will choose

$$
c_{+}=\frac{1}{\sqrt{2}}, \quad c_{-}=\frac{i}{\sqrt{2}}
$$

Define

$$
\psi_{ \pm}\left[Q, \Phi ; \tau_{0}\right] \equiv\left\langle Q, \Phi \mid \pm \alpha, 0_{M}\right\rangle .
$$

Since $| \pm \alpha\rangle$ and $\left|0_{M}\right\rangle$ are Gaussian states, the RDM of the detector must have the form

$$
\rho^{R}\left(Q, Q^{\prime} ; \tau\right)=\left|c_{+}\right|^{2} \rho_{++}+\left|c_{-}\right|^{2} \rho_{--}+c_{+}^{*} c_{-} \rho_{+-}+c_{+} c_{-}^{*} \rho_{-+},
$$

where

$$
\begin{aligned}
\rho_{ \pm \pm}\left(Q, Q^{\prime} ; \tau\right) & =\int \mathcal{D} \Phi \psi_{ \pm}[Q, \Phi ; \tau] \psi_{ \pm}^{*}\left[Q^{\prime}, \Phi ; \tau\right] \\
& =\exp \left\{-G^{i j}(\tau) Q_{i} Q_{j} \pm J_{+}^{i}(\tau) Q_{i}+F_{ \pm \pm}(\tau)\right\}, \\
\rho_{ \pm \mp}\left(Q, Q^{\prime} ; \tau\right) & =\int \mathcal{D} \Phi \psi_{ \pm}[Q, \Phi ; \tau] \psi_{\mp}^{*}\left[Q^{\prime}, \Phi ; \tau\right] \\
& =\exp \left\{-G^{i j}(\tau) Q_{i} Q_{j} \mp J_{-}^{i}(\tau) Q_{i}+F_{ \pm \mp}(\tau)\right\} .
\end{aligned}
$$

The coefficients $G^{i j}$ are given by (21)-(23), and

$$
\begin{aligned}
J_{ \pm}^{1}+J_{ \pm}^{2} & =2 \frac{\langle Q\rangle_{ \pm}}{\left\langle Q^{2}\right\rangle_{00}} \\
J_{ \pm}^{1}-J_{ \pm}^{2} & =\frac{2 i}{\hbar}\left[\langle P\rangle_{ \pm}-\frac{\langle P, Q\rangle_{00}}{\left\langle Q^{2}\right\rangle_{00}}\langle Q\rangle_{ \pm}\right]
\end{aligned}
$$

where

$$
\langle\mathcal{O}\rangle_{ \pm} \equiv \frac{\operatorname{Tr}\left[\hat{\mathcal{O}} \rho_{ \pm+}\right]}{\operatorname{Tr} \rho_{ \pm+}}
$$

The normalization conditions give

$$
\begin{aligned}
& e^{F_{++}}=e^{F_{--}}=\frac{\operatorname{Tr} \rho_{++}}{\sqrt{2 \pi\left\langle Q^{2}\right\rangle_{00}}} \exp \frac{-\langle Q\rangle_{+}^{2}}{8\left\langle Q^{2}\right\rangle_{00}}, \\
& e^{F_{-+}}=e^{F_{+-}}=\frac{\operatorname{Tr} \rho_{-+}}{\sqrt{2 \pi\left\langle Q^{2}\right\rangle_{00}}} \exp \frac{-\langle Q\rangle_{-}^{2}}{8\left\langle Q^{2}\right\rangle_{00}},
\end{aligned}
$$

with $\operatorname{Tr} \rho_{++}=1$ and $\operatorname{Tr} \rho_{-+}=e^{-m_{0} \Omega_{r} q_{0}^{2} / \hbar}$.

For $c_{+}=1 / \sqrt{2}$ and $c_{-}=i / \sqrt{2}$, the purity reads

$$
\begin{aligned}
& \mathcal{P}=\frac{\hbar}{4 \mathcal{U}}\left\{1+\exp \left[-\frac{\left\langle Q^{2}\right\rangle_{00}}{\mathcal{U}^{2}}\left(\langle P\rangle_{+}-\frac{\langle P, Q\rangle_{00}}{\left\langle Q^{2}\right\rangle_{00}}\langle Q\rangle_{+}\right)^{2}-\frac{\langle Q\rangle_{+}^{2}}{\left\langle Q^{2}\right\rangle_{00}}\right]+\right. \\
& \left.\left(\exp \left[-\frac{\left\langle Q^{2}\right\rangle_{00}}{\mathcal{U}^{2}}\left(\langle P\rangle_{-}-\frac{\langle P, Q\rangle_{00}}{\left\langle Q^{2}\right\rangle_{00}}\langle Q\rangle_{-}\right)^{2}-\frac{\langle Q\rangle_{-}^{2}}{\left\langle Q^{2}\right\rangle_{00}}\right]-1\right) e^{-\frac{2}{\hbar} m_{0} \Omega_{r} q_{0}^{2}}\right\}
\end{aligned}
$$



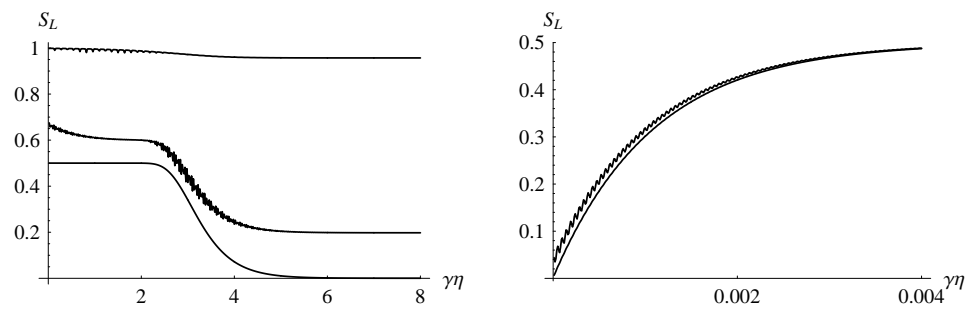

FIG. 5: Evolution of linear entropies $S_{L}=1-\mathcal{P}$ of (C15) with different parameters. (Left) $\gamma=10^{-7}$ (upper curve), $10^{-4}$ (middle) and 0.1 (lower), $q_{0}=10, \Omega=2.3, a=0, m_{0}=\hbar=1, \Lambda_{1}=\Lambda_{0}=10000$. One can compare the lower curve with FIG.2 in Ref. 34]. (Right) $\gamma=10^{-7}, \Lambda_{1}=\Lambda_{0}=100$ (lower curve) and 2000(upper), with other parameters the same as before. This plot shows that the growing rate of $S_{L}$ right after the coupling is switched on is almost independent of the UV cut-off in the ultraweak coupling limit.

In the Heisenberg picture, it is easy to obtain [1]

$$
\begin{aligned}
& \langle Q\rangle_{ \pm}=\frac{\langle\alpha|\hat{Q}(\tau)| \pm \alpha\rangle}{\langle\alpha \mid \pm \alpha\rangle}=\frac{q_{0}}{2}\left(q^{a *} \pm q^{a}\right), \\
& \langle P\rangle_{ \pm}=\frac{\langle\alpha|\hat{P}(\tau)| \pm \alpha\rangle}{\langle\alpha \mid \pm \alpha\rangle}=m_{0} \frac{q_{0}}{2}\left(\dot{q}^{a *} \pm \dot{q}^{a}\right),
\end{aligned}
$$

where $q^{a}$ has been given in (32). Evolutions of linear entropies $S_{L}=1-\mathcal{P}$ with different parameters have been illustrated in FIG. 5 .

In the ultraweak coupling limit, the purity (C15) becomes

$$
\begin{aligned}
\mathcal{P} \approx & \frac{1}{2}\left(1+\exp \left[-\frac{2}{\hbar} m_{0} \Omega q_{0}^{2} e^{-2 \gamma \tau}\right]\right)-\frac{1}{2} e^{-2 m_{0} \Omega q_{0}^{2} / \hbar} \\
& +\frac{1}{2} \exp \left[\frac{2}{\hbar} m_{0} \Omega q_{0}^{2}\left(e^{-2 \gamma \tau}-1\right)\right] .
\end{aligned}
$$

The second line decays from $1 / 2$ to 0 in a time scale $\tau_{\text {decoh }} \sim 1 / \gamma m_{0} \Omega q_{0}^{2}$, while the first line grows from $1 / 2$ to 1 in a time scale $\tau_{\text {recoh }}=(2 \gamma)^{-1} \ln \left(2 m_{0} \Omega q_{0}^{2} / \ln 2\right)$.

When $m_{0} \Omega q_{0}^{2}$ is large, $\tau_{\text {recoh }} \gg \tau_{\text {decoh }}$, so after the coupling is switched on, the purity drops from 1 to $1 / 2$ rapidly in a time scale $\tau_{\text {decoh }}$, stay at $1 / 2$ for a relatively long time scale $\tau_{\text {recoh }}$, then goes back to 1 slowly. The evolution of the linear entropy in this limit has been shown in FIG. 5 (the lower curve in the left plot), which looks virtually the same as the "entropy of oscillator" in FIG. 2 of Ref. [34].

The recoherence occurs at $\eta \approx \tau_{\text {recoh }}$, when the separation between the two wave packets become comparable to the width of each wave packet $\left(q_{0} e^{-\gamma \tau_{\text {recoh }}}=\sqrt{\left\langle Q^{2}\left(\tau_{\text {recoh }}\right)\right\rangle}=\sqrt{\hbar / m_{0} \Omega}\right.$. $)$ Before $\tau_{\text {recoh }}$ the two coherent states $\varphi_{ \pm}(Q ; \tau)$ with $\varphi_{ \pm}\left(Q ; \tau_{0}\right)=\langle Q \mid \pm \alpha\rangle$ are nearly orthogonal to each other and from (C9) and (C14) $\rho_{+-}$and $\rho_{-+}$are suppressed so the RDM looks like $\rho^{R}\left(Q, Q^{\prime}\right) \approx(1 / 2) \varphi_{+}(Q) \varphi_{+}^{*}\left(Q^{\prime}\right)+(1 / 2) \varphi_{-}(Q) \varphi_{-}^{*}\left(Q^{\prime}\right)$, which yields $\mathcal{P} \approx 1 / 2$.

Note that, unlike the time scales in (40) and (49), here $\tau_{\text {decoh }}$ and $\tau_{\text {recoh }}$ depend on $m_{0}$ and $\Omega$ in addition to $\gamma$.

In [34] the authors reported that the entropy initially grows in a decoherence time corresponding to the cut-off time scale, even though they were working in the weak coupling limit. On this point we disagree with them since our $\tau_{\text {decoh }} \sim 1 / \gamma m_{0} \Omega q_{0}^{2}$ is virtually independent of any cut-off in this limit [see also FIG. 5 (right)].

Beyond the ultraweak coupling limit, the linear entropy is always close to 1 and never decays to 0 , so the late-time recoherence never occurs in non-Markovian regime or the ultrahigh acceleration regime (the upper and middle curves in FIG. 5 (left)). Again the late-time value of the purity of the detector is (35), quite independent of the initial states of the detector.

[1] S.-Y. Lin and B. L. Hu, Phys. Rev. D 73, 124018 (2006) gr-qc/0507054.

[2] S.-Y. Lin and B. L. Hu, Phys. Rev. D 76, 064008 (2007) gr-qc/0611062.

[3] T. Yu and J. H. Eberly, Phys. Rev. Lett. 93, 140404 (2004).

[4] T. Yu and J. H. Eberly, Phys. Rev. B 66193306 (2002).

[5] T. Yu and J. H. Eberly, Phys. Rev. B 68, 165322 (2003). 
[6] B. Reznik, A. Retzker and J. Silman, Phys. Rev. A 71042104 (2005).

[7] A. Retzker, J. I. Cirac and B. Reznik, Phys. Rev. Lett. 94050504 (2005).

[8] Z. Ficek and R. Tanas, Phys. Rev. A 74, 024304 (2006).

[9] Z. Ficek and R. Tanas, Phys. Rep. 372369 (2002).

[10] Ch. Anastopoulos, S. Shresta and B. L. Hu, "Quantum Entanglement under Non-Markovian Dynamics of Two Qubits Interacting with a common Electromagnetic Field", quant-ph/0610007

[11] G. L. Ver Steeg and N. Menicucci, "Entangling Power of an Expanding Universe" arXiv:0711.3066.

[12] C. H. Chou, T. Yu and B. L. Hu, Phys. Rev. E 77011112 (2008).

[13] C. H. Chou, B. L. Hu and T. Yu, Physica A387 432 (2008).

[14] W. K. Wootters, Phys. Rev. Lett. 80, 2245 (1998).

[15] H. Barnum, E. Knill, G. Ortiz, R. Somma, and L. Viola, Phys. Rev. Lett. 92, 107902 (2004).

[16] H. Barnum, E. Knill, G. Ortiz, and L. Viola, Phys. Rev. A 68, 032308 (2003).

[17] J. D. Bekenstein, Phys. Rev. D 7, 2333 (1973).

[18] J. D. Bekenstein, in The Seventh Marcel Grossmann Meeting on recent developments in theoretical and experimental general relativity, gravitation, and relativistic field theories : Proceedings, edited by R. T. Jantzen, G. Mac Keiser, and R. Ruffini (World Scientific, Singapore, 1996) gr-qc/9409015.

[19] S. W. Hawking, Nature 248, 30 (1974).

[20] S. W. Hawking, Commun. Math. Phys. 43, 199 (1975).

[21] S. W. Hawking, Phys. Rev. D 13, 191 (1976).

[22] S. W. Hawking, Phys. Rev. D 14, 2460 (1976).

[23] S. W. Hawking, Phys. Rev. D 72, 084013 (2005).

[24] D. N. Page, in Proceedings of the 9th International Conference on General Relativity and Gravitation, edited by E. Schmutzer (Friedrich Schiller University, Jena, 1980).

[25] J. Preskill, in Black Holes, Membranes, Wormholes and Superstrings, edited by K. Kalara and D. V. Nanopoulos (World Scientific, Singapore, 1993) hep-th/9209058.

[26] D. N. Page, in Proceedings of 5th Canadian Conference on General Relativity and Relativistic Astrophysics, edited by R. B. Mann and R. G. McLenaghan (World Scientific, Singapore, 1994) hep-th/9305040.

[27] E. Witten, Phys. Rev. D 44, 314 (1991).

[28] C. G. Callan, S. B. Giddings, J. A. Harvey, and A. Strominger, Phys. Rev. D 45, R1005 (1992).

[29] J. G. Russo, L. Susskind, and L. Thorlacius, Phys. Lett. B292, 13 (1992).

[30] J. G. Russo, L. Susskind, and L. Thorlacius, Phys. Rev. D 46, 3444 (1992).

[31] C. Holzhey and F. Wilczek, Nucl. Phys. B380, 447 (1992).

[32] F. Wilczek, in Black Holes, Membranes, Wormholes and Superstrings, edited by K. Kalara and D. V. Nanopoulos (World Scientific, Singapore, 1993) hep-th/9302096.

[33] J. Polchinski and A. Strominger, Phys. Rev. D 50, 7403 (1994).

[34] J. R. Anglin, R. Laflamme, W. H. Zurek and J. P. Paz, Phys. Rev. D 52, 2221 (1995).

[35] B. L. Hu, Erice Lectures, Sept. 1995, in String Gravity and Physics at the Planck Energy Scale, edited by N. Sanchez and A. Zichichi (Kluwer, Dordrecht, 1996) gr-qc/9511075.

[36] D. A. Lowe and L. Thorlacius, Phys. Rev. D 73, 104027 (2006).

[37] S. B. Giddings, Phys. Rev. D 74, 106005 (2006).

[38] D. R. Terno, Int. J. Mod. Phys. D 142307 (2005).

[39] J. A. Smolin and J. Oppenheim, Phys. Rev. Lett. 96, 081302 (2006).

[40] S. L. Braunstein and A. K. Pati, Phys. Rev. Lett. 98, 080502 (2007).

[41] A. Raval, B. L. Hu and Don Koks, Phys. Rev. D 55, 4795 (1997).

[42] B. L. Hu and P. R. Johnson, "Beyond Unruh Effect: Nonequilibrium Quantum Dynamics of Moving Charges". Invited Talk at the Capri Workshop on Quantum aspects of Beam Physics, Oct. 2000. Proceedings edited by Pisin Chen. (WorldScientific, Singapore, 2001) quant-ph/0012132

[43] W. G. Unruh, Phys. Rev. D14, 870 (1976).

[44] B. S. DeWitt, in General Relativity: an Einstein Centenary Survey, edited by S. W. Hawking and W. Israel (Cambridge University Press, Cambridge, 1979).

[45] N. D. Birrell and P. C. W. Davies, Quantum Fields in Curved Space (Cambridge University Press, Cambridge, 1982).

[46] A. Peres, Quantum Theory: Concepts and Methods (Kluwer, Dordrecht, 1995).

[47] C. Cohen-Tannudji, J. Dupont-Roc and G. Grynberg, Atom-Photon Interactions (Wiley, New York, 1992).

[48] G. Vidal, J. Mod. Opt. 47355 (2000).

[49] I. Bengtsson and K. Życzkowski Geometry of Quantum States. An Introduction to Quantum Entanglement (Cambridge University Press, Cambridge, 2006)

[50] J. D. Bekenstein, Phys. Rev. D 12, 3077 (1975).

[51] J. D. Bekenstein and A. Meisels, Phys. Rev. D 15,2775 (1976).

[52] J. D. Bekenstein, in Prodeedings of the Eight Marcel Grossmann Meeting, edited by T. Piran and R. Ruffini (World Scientific, Singapore, 1999) gr-qc/9710076.

[53] J. D. Bekenstein and G. Gour, Phys. Rev. D 66024005 (2002).

[54] Note that as different from atoms, for a real black hole its event horizon will have an effect on such emission. 